\title{
Statin Therapy to Revert Hypercoagulability and Prevent Venous Thromboembolism: A Narrative Review
}

\author{
${ }^{1}$ Department of Clinical Pathology, School of Medical Sciences, \\ University of Campinas (UNICAMP), Campinas, Brazil \\ 2 Department of Clinical Epidemiology, Leiden University Medical \\ Center, Leiden, The Netherlands \\ ${ }^{3}$ Einthoven Laboratory for Experimental Vascular Medicine, Leiden \\ University Medical Center, Leiden, The Netherlands \\ ${ }^{4}$ Department of Internal Medicine, Section of Thrombosis and Hemostasis, \\ Leiden University Medical Center, Leiden, The Netherlands
}

Fernanda A. Orsi, MD, PhD ${ }^{1,2}$ Suzanne C. Cannegieter, MD, PhD²,3,4 Willem M. Lijfering, MD, $\mathrm{PhD}^{2,3}$

Semin Thromb Hemost 2019;45:825-833.

\author{
Address for correspondence Fernanda A. Orsi, MD, PhD, Rua Tessália \\ Vieira de Camargo, 126. Campinas, SP., Brazil \\ (e-mail: ferorsi@unicamp.br).
}

\author{
Abstract \\ Keywords \\ - blood coagulation \\ - clinical trials \\ - hydroxymethyl- \\ glutaryl-CoA \\ reductase inhibitors \\ - prevention \\ - venous thrombosis
}

Venous thromboembolism (VTE) causes a major disease burden worldwide, so that effective preventive measures are warranted. Although oral anticoagulation is effective in preventing VTE episodes, bleeding complications are a major concern that may lead to treatment avoidance. Statin therapy, which is widely used for prevention of arterial cardiovascular disease, is a promising alternative treatment for VTE prophylaxis, as the drug may affect hemostasis without increasing the risk of bleeding. In the past years, clinical studies have suggested that statins can interfere with blood coagulation and, in turn, reduce the risk of VTE. These effects, however, are still regarded with skepticism, as the underlying mechanisms by which statins may affect hemostasis in humans are not clear and data showing that statin therapy reduces VTE risk mostly came from observational studies, while only one randomized trial was conducted to evaluate this issue. In this review, the authors summarize the currently available evidence regarding the effect of statin therapy on coagulation and on VTE prevention. Recent randomized data showed that statin therapy, in particular rosuvastatin, leads to decreased levels of coagulation factors in patients with prior VTE. This evidence provides a reasonable basis for interventional studies necessary to establish the efficacy of statins on reducing the risk of incident and recurrent VTE.
Venous thromboembolism (VTE) is a condition that comprises deep vein thrombosis (DVT), pulmonary embolism $(\mathrm{PE})$ or both. The reported annual incidence of VTE ranges from 7.5 to 26.9 per 10,000 individuals in the population, with the highest rates being reported in the elderly. ${ }^{1}$ In addition, VTE mortality rates range from 0.94 to 3.23 per 10,000 individuals per year, varying across countries. ${ }^{2}$ Due to the high incidence rates, associated disabilities and mortality, VTE causes a major disease burden worldwide, ${ }^{1}$ which underscores the necessity of effective preventive measures and adequate treatment.

Approximately 50 to $60 \%$ of VTE episodes are provoked by major risk factors, such as cancer, surgery, immobilization, estrogen therapy, and pregnancy. Cases in which a transient or a persistent risk factor cannot be identified are categorized as unprovoked VTE. ${ }^{3,4}$ Because the risk of recurrent VTE is higher after an unprovoked event compared with a provoked one, categorizing VTE episodes is important to determine the duration of anticoagulation treatment. ${ }^{5}$ After anticoagulation therapy is discontinued, the risk of VTE recurrence is up to $10 \%$ per year in patients with unprovoked VTE, ${ }^{6}$ and extended use of oral anticoagulants is associated with a sevenfold decrease in the risk of recurrent VTE. ${ }^{7,8}$ However, prolonged oral anticoagulant therapy increases by two- to threefold the risk of having a clinically significant bleeding episode compared with placebo, ${ }^{7,8}$ which may result in treatment avoidance. Therefore,
Issue Theme Recent Advances in Thrombosis and Hemostasis-Part V; Guest Editor: Sam Schulman, MD, PhD.
Copyright @ 2019 by Thieme Medical Publishers, Inc., 333 Seventh Avenue, New York, NY 10001, USA. Tel: +1(212) 584-4662.
DOI https://doi.org/ 10.1055/s-0039-1687911. ISSN 0094-6176. 
the search for new medications capable of decreasing VTE risk without increasing the risk of bleeding is warranted.

In this context, statins could be an alternative medication for VTE prophylaxis because they may have antithrombotic effect without causing bleeding complications. ${ }^{9}$ Available evidence from clinical trials consistently indicates that statins do not increase the risk of bleeding. Although results from the Stroke Prevention by Aggressive Reduction in Cholesterol Levels (SPARCL) trial $^{10}$ indicated that atorvastatin at $80 \mathrm{mg}$ per day was associated with a $66 \%$ increase in the risk of hemorrhagic stroke compared with placebo (hazard ratio, 1.66; 95\% confidence interval $[\mathrm{CI}]: 1.08-2.55)$, these observations were not confirmed in other trials. ${ }^{11-14}$ This suggests that results from the SPARCL trial were due to a type I error. In the Heart Protection Study, ${ }^{11}$ no difference in hemorrhagic stroke was observed between simvastatin at $40 \mathrm{mg}$ per day and placebo (incidence rate ratio, 0.95; 95\% CI: 0.65-1.40). The Justification for the Use of Statins in Prevention: An Intervention Trial Evaluating Rosuvastatin (JUPITER) trial found no difference between rosuvastatin at $20 \mathrm{mg}$ per day and placebo in the rates of intracranial hemorrhage ${ }^{12}$ or other bleeding episodes. ${ }^{13}$ A meta-analysis of randomized trials and observational studies ${ }^{14}$ demonstrated that statins were not associated with an increased risk of intracerebral hemorrhage. The pooled risk ratio for bleeding on statin in randomized trials was 1.10 (95\% CI: 0.86-1.41) and was 0.94 (95\% CI: 0.81-1.10) in cohort studies. Finally, the American Heart Association/American Stroke Association guidelines ${ }^{15}$ state that there is insufficient data to recommend restrictions on use of statin agents for the management of intracerebral hemorrhage. In addition, longterm use of statins, which is required for prophylactic treatments, is well tolerated and side effects are manageable. ${ }^{16}$

Statins are widely used in patients with cardiovascular risk factors for prophylaxis of cardiovascular disease (CVD). ${ }^{17}$ Since CVD and VTE have some common risk factors, such as older age, male sex, smoking history, sedentary lifestyle, obesity, and hypercoagulability, ${ }^{18-21}$ statins are frequently recommended anyway to patients requiring VTE prophylaxis. Thus, the possibility of using one single drug to prevent arterial CVDs and VTE would diminish the pill burden associated with the use of several classes of drugs. Therefore, statin therapy could become an alternative treatment for VTE prophylaxis if statins are proven to downregulate hemostasis and prevent VTE episodes.

In this article, evidence of association between statins, hemostasis, and VTE risk will be reviewed. For this purpose, we searched MEDLINE electronic databases to select the manuscripts. The searches combined the MESH terms related to the intervention (i.e., hydroxymethylglutaryl-CoA reductase inhibitors) and outcomes (i.e., hemostasis, blood coagulation, VTE, embolism, and thrombosis). Basic science studies and clinical studies, particularly meta-analysis and systemic reviews, were selected for this review.

\section{The Effect of Statins on Hemostasis}

Statins are a class of drugs that decrease the serum levels of cholesterol through the inhibition of the enzyme 3-hydroxy-3methylglutaryl coenzyme A reductase. By inhibiting this enzyme, statins reduce the hepatocyte cholesterol content, thus promoting enhanced expression of low-density lipoprotein (LDL) receptors on the cell membrane. The increased expression of this receptor leads to increased receptor-mediated endocytosis of LDL and, in turn, to decreased serum levels of LDL. $^{22}$

Statin use decreases the risk of CVD not only because of the lipid-lowering effect but also because of an anti-inflammatory effect. ${ }^{23}$ Results from the JUPITER trial demonstrated that the risk of CV events decreased by $79 \%$ in participants who achieved the targets of high-sensitivity C-reactive protein less than $1 \mathrm{mg} / \mathrm{L}$ and LDL cholesterol less than $1.8 \mathrm{mmol} / \mathrm{L}$ (hazard ratio $0.21,95 \% \mathrm{CI}: 0.09-0.51$ ). On the other hand, the effect of rosuvastatin on decreasing the risk of CV events was less prominent in patients who achieved only the target of LDL cholesterol (hazard ratio $0.49,95 \% \mathrm{CI}: 0.37-0.66$ ). These results suggest that both lipid lowering and anti-inflammatory effects are important for the prevention of CV events. ${ }^{23}$

Besides the lipid lowering and the anti-inflammatory effects, statins are alleged to have pleiotropic effect on the process of hemostasis. Basic research and clinical studies have investigated whether statins can interfere with platelet activity, coagulation, thrombin generation, and fibrinolysis. These studies will be reviewed in this topic.

\section{Effects of Statins on Platelet Activity}

Studies in vitro and in animal models have demonstrated that statins can decrease platelet activation by several mechanisms. Rosuvastatin was shown to inhibit platelet degranulation in rat models of myocardial infarction ${ }^{24}$ and atorvastatin to decrease platelet activation and upregulate the synthesis of nitric oxide (NO) by platelets and endothelial cells in mice models of cerebral ischemia. ${ }^{25-27}$ Pravastatin was shown to suppress platelet-dependent procoagulant activity in vitro by decreasing the expression of P-selectin and the deposition of tissue factor (TF), thrombin, and fibrin on adherent platelets. ${ }^{28,29}$

The first clinical study that evaluated the effects of statins on platelet activity enrolled patients with hypercholesterolemia, and showed that pravastatin could affect platelet aggregation and platelet-dependent thrombin generation in association with reduced serum cholesterol levels. ${ }^{30}$ Atorvastatin, simvastatin, fluvastatin, and cerivastatin were also shown to reduce Pselectin levels ${ }^{31}$ and platelet aggregation with different agonists, such as arachidonic acid, ${ }^{32,33}$ adenosine diphosphate, collagen, and epinephrine. ${ }^{34,35}$ Conversely, the discontinuation of statins led to increased platelet activity. ${ }^{33}$ In patients with hypercholesterolemia, the effect of statins on platelet aggregation seemed to occur in association with increased platelet-derived NO release, in a dose-dependent manner and independently of cholesterol-lowering effect. ${ }^{34}$ In healthy individuals, use of simvastatin for 7 days led to a reduction in platelet aggregation triggered by arachidonic acid. ${ }^{36}$ Although these studies point to an effect of statins on platelet activity, most of the data came from nonrandomized studies, and therefore these results may be biased by confounding or due to regression to the mean.

In the STAtins Reduce Thrombophilia (START) trial, in which individuals with prior VTE were randomized to receive either rosuvastatin at $20 \mathrm{mg}$ daily for 28 days or no 
intervention, rosuvastatin use had no effect on platelet activation mediated by thromboxane $\mathrm{A}_{2}\left(\mathrm{TxA}_{2}\right) .{ }^{37}$ Although the observations from this randomized controlled trial (RCT) do not confirm that rosuvastatin decreases platelet activation mediated by $\mathrm{TxA}_{2}$, it is not possible to exclude potential other antiplatelet effects of rosuvastatin since other platelet function assays were not performed.

\section{Effect of Statins on Coagulation}

Evidence with regard to the effect of statins on coagulation was first demonstrated in the late 1990s and early 2000s. The first studies, performed in vitro, demonstrated that both simvastatin and fluvastatin were capable of reducing TF gene expression in human monocytes, endothelial cells, and smooth muscle cells in a dose-dependent manner. ${ }^{38,39}$ Not only TF was suppressed but also thrombin formation could be inhibited by simvastatin in vitro. ${ }^{40}$ Lovastatin, simvastatin, and mevastatin were shown, in vitro, to enhance the activated protein C-mediated suppression of thrombin generation, in part via increased levels of thrombomodulin, in a concentration-dependent manner, that is dependent on inhibition of the Rho/Rho-kinase pathway. ${ }^{41}$ Later on, the inhibitory effect of statins on TF expression was replicated in vivo. Studies in hyperlipidemic mice models showed that statins diminished the expression of TF in atherosclerotic lesions and monocytes, ${ }^{42}$ independently of the reduction in plasma lipids. ${ }^{43,44}$ The mechanisms of statin-induced TF inhibition involved a direct inhibitory effect on Rho/Rho-kinase pathway and, in turn, TF expression. ${ }^{39,45}$ This lipid-independent mechanism was further confirmed in a study with a atherosclerotic rabbit model showing that fluvastatin directly interfered with the transcriptional activation of TF gene, by downregulating the nuclear factor kappa-light-chain-enhancer of activated B cells (NF-kB) pathway in endothelial cells. ${ }^{46}$ Therefore, basic research studies consistently demonstrated that statins can downregulate TF expression $38,39,42$ through a direct pathway, independent of the lipid-lowering mechanism. ${ }^{45,46}$

Clinical trials have also demonstrated that statin therapy, either with simvastatin, ${ }^{47}$ atorvastatin ${ }^{48-50}$ or cerivastatin, ${ }^{35,51}$ affects coagulation factors and thrombin generation, although the mechanisms behind these effects are less clear. The first study, published in $1997,{ }^{52}$ showed that simvastatin treatment decreased plasma levels of prothrombin fragment $\mathrm{F} 1+2(\mathrm{~F} 1+2)$ by $35 \%$ and monocyte TF antigen and activity by 68 and 61\%, respectively, in hypercholesterolemic patients. The effect of simvastatin and cerivastatin in reducing tissue factor pathway inhibitor (TFPI) and factor (F) VII was also demonstrated in crossover studies ${ }^{35,51}$ and in a small randomized trial. ${ }^{53}$ In an RCT aimed to compare atorvastatin with placebo in patients eligible for two-step carotid endarterectomy (CEA), atorvastatin reduced TF and TFPI antigen levels in blood (29 and 18\% reduction, respectively), and TF activity (56\% reduction) in plaques removed at the second CEA, as compared with placebo. ${ }^{48}$ Further clinical studies demonstrated that 12 weeks of atorvastatin therapy could lead to decreased FVII levels and activity, ${ }^{49,50}$ coinciding with a decrease in the serum levels of LDL-cholesterol (LDL-C), very low-density lipoprotein cholesterol (VLDL-C), and triglycer- ides. $^{32}$ The effect of statin on TF was also evaluated in individuals without dyslipidemia or CVD. In a study of experimental endotoxemia, 20 healthy men were randomized to receive either simvastatin $(80 \mathrm{mg} / \mathrm{d})$ or placebo for 4 days before intravenous administration of lipopolysaccharides (LPS). Simvastatin premedication attenuated the increase in monocyte TF expression and reduced the formation of $\mathrm{F} 1+2$ in response to LPS without affecting platelet aggregation. ${ }^{54}$

The effect of statin therapies on FV, ${ }^{55-57}$ von Willebrand factor (VWF), ${ }^{55,58,59}$ and natural anticoagulants ${ }^{59}$ has also been reported in several studies. ${ }^{55-59}$ Two small clinical studies performed in patients with $\mathrm{CVD}^{56,57}$ showed that early simvastatin therapy decreased FVa generation and increased FVa inactivation by protein $\mathrm{C}$ in blood samples collected from sites of microvascular injury (bleeding-time wounds). Simvastatin and pravastatin reduced VWF levels in patients with hypercholesterolemia after 3 months of therapy. In a clinical trial, 45 patients with unstable angina were randomized to receive either atorvastatin or placebo for 6 weeks. Levels of coagulation markers, such as tissue plasminogen activator (tPA) levels, protein $\mathrm{C}$, protein S, FV, FVII, and VWF, were measured after 1 and 6 weeks with treatment and compared with baseline. ${ }^{55}$ In the placebo arm, all coagulation markers, except for protein $\mathrm{S}$, increased from baseline to the end of the first week with treatment. At 6 weeks, protein $C$ and antithrombin remained elevated in patients receiving placebo, when compared with baseline. These parameters did not change substantially in patients who received atorvastatin, suggesting that atorvastatin could regulate the levels of liver-derived coagulation markers. ${ }^{55}$ Furthermore, in a randomized trial conducted with 60 patients with acute coronary syndrome, both atorvastatin low $(10 \mathrm{mg})$ and high $(80 \mathrm{mg})$ dose prevented elevation of VWF from baseline to the end of the first week with treatment, when compared with placebo. ${ }^{59}$ After 2 weeks with treatment, levels of VWF were similar between both treatment arms and placebo. ${ }^{59}$ Besides the reduction in some coagulation factors, simvastatin, ${ }^{47,51,56,60}$ pravastatin, $^{58}$ and atorvastatin 61,62 therapies were also shown to reduce the thrombin generation potential and the level of D-dimer, ${ }^{63}$ both markers of hypercoagulability. In the Long-Term Intervention with Pravastatin in Ischaemic Disease (LIPID) study, 9,014 patients were randomized to pravastatin $40 \mathrm{mg}$ or placebo for 3 to 36 months after an acute coronary syndrome. After 1 year of treatment, Ddimer levels in placebo group did not change from baseline (mean change of $1 \mathrm{ng} / \mathrm{mL}$ ), while D-dimer levels in patients receiving pravastatin decreased significantly (mean change $-12 \mathrm{ng} / \mathrm{mL}$ ). The difference between groups in absolute change of D-dimer levels from baseline to the end of the first year of the study was statistically different (mean levels at year 1 were 172 $\mathrm{ng} / \mathrm{mL}$ in placebo arm and $166 \mathrm{ng} / \mathrm{mL}$ in patients receiving pravastatin). ${ }^{64}$

It is noteworthy that most of these data came from nonrandomized studies in which confounding and regression to the mean cannot be ruled out. Stronger evidence that statins can affect coagulation came from two meta-analyses of randomized trials. ${ }^{65,66}$ These meta-analyses demonstrated a significant decrease in the plasma levels of VWF (standardized mean difference [SMD] $-0.54,95 \% \mathrm{CI}$ : -0.87 
to -0.21$)^{65}$ and D-dimer (SMD $-0.988 \mu \mathrm{g} / \mathrm{mL}, 95 \% \mathrm{CI}$ : -1.590 to -0.385$)^{66}$ following statin therapy. In both studies, the effects on VWF and D-dimer levels were more evident after atorvastatin or simvastatin therapy was commenced, when compared with no statin use. Limitations of the two metaanalyses are the inclusion of heterogeneous patient populations and treatment assignments and, according to funnel plot analyses, the possibility of publication bias.

Evidence of the effect of statin therapy on coagulation factors recently came from the START trial, in which patients with a prior VTE were randomized to rosuvastatin treatment or nonstatin. ${ }^{67}$ The mean FVIII:C levels decreased $7.2 \mathrm{IU} / \mathrm{dL}$ (95\% CI: 2.9-11.5) in rosuvastatin users from baseline to the end of treatment, while among nonusers, no change in FVIII: C was observed (mean difference $-0.1 ; 95 \% \mathrm{CI}$ : -3.0 to 2.9 ). Rosuvastatin therapy also decreased the levels of FVII:C (mean change $-3.6 \mathrm{IU} / \mathrm{dL}$; $95 \% \mathrm{CI}:-0.2$ to -7.1 ) and FXI:C (mean change $-5.9 \mathrm{IU} / \mathrm{dL} ; 95 \% \mathrm{CI}$ : -2.7 to -9.0 ) from baseline to the end of the study. Subgroup analyses revealed that the effect of rosuvastatin on decreasing the levels of coagulation factors was more pronounced in participants with unprovoked VTE or with cardiovascular risk factors. ${ }^{67}$ The results are illustrated in -Fig. $\mathbf{1}$.

\section{Effect of Statins on Fibrinolysis}

Clinical studies that evaluated the effect of statins on fibrinolysis have presented controversial results. In a study with 46 patients with coronary artery disease randomized to receive either placebo or fluvastatin, patients receiving fluvastatin had a decrease in tPA and an increase in plasminogen activator inhibitor-1 (PAI-1) activity. ${ }^{68}$ Pravastatin decreased $\mathrm{TPA}^{69}$ and PAI- ${ }^{69,70}$ levels by 10 to $20 \%$ among hyperlipidemic patients, accompanied by a decrease in LDLC levels. Atorvastatin also increased the levels of PAP by $50 \%$, decreased PAI-1 activity by $34 \%$, and decreased plateletdependent thrombin generation by $48 \%$ in hyperlipidemic patients. ${ }^{71}$ A shift to a profibrinolytic profile related to statin therapy was also observed in sets of patients with coronary disease undergoing coronary artery bypass grafting, ${ }^{72}$ in hyperlipidemic women, ${ }^{73}$ in patients with essential hypertension, ${ }^{74}$ and in patients with chronic obstructive pulmonary disease. ${ }^{75}$ In healthy individuals, a large cohort study showed that participants using statins had lower levels of Ddimer and FVIII and higher levels of fibrinogen and PAI-1, in comparison with nonstatin users. ${ }^{76}$ These results, however, seem contradictory since lower D-dimer and FVIII suggest a decrease in hemostatic activity, while higher fibrinogen and PAI-1 levels suggest hypercoagulability.

The strongest evidence for the effect of statin therapy on fibrinolysis came from a recent meta-analysis of RCTs, where the pooled analysis revealed that statin therapy reduced plasma levels of PAI-1compared with no statin therapy. ${ }^{77}$ Although these findings suggest that statins stimulate fibrinolysis, result could also be plagued by publication bias. No additional evidence from RCTs of the effect of statin therapy on fibrinolysis has been published recently.

\section{The Importance of the Lipid-Lowering Effect on Hemostasis}

Basic research studies have consistently shown that the effect of statins on hemostasis is independent of the drug-related lipid-lowering effect. Studies in vitro have confirmed that statins can directly inhibit the Rho/Rho-kinase pathway and consequently NF-kB activity and TF expression in monocytes and endothelial cells. ${ }^{39,40,45,46}$ Such mechanisms of action are different from that involved in the lipid-lowering process. ${ }^{22}$

Additionally, studies in hyperlipidemic mice and rabbits have shown that statins can decrease the expression of TF in

\section{Unprovoked VTE}

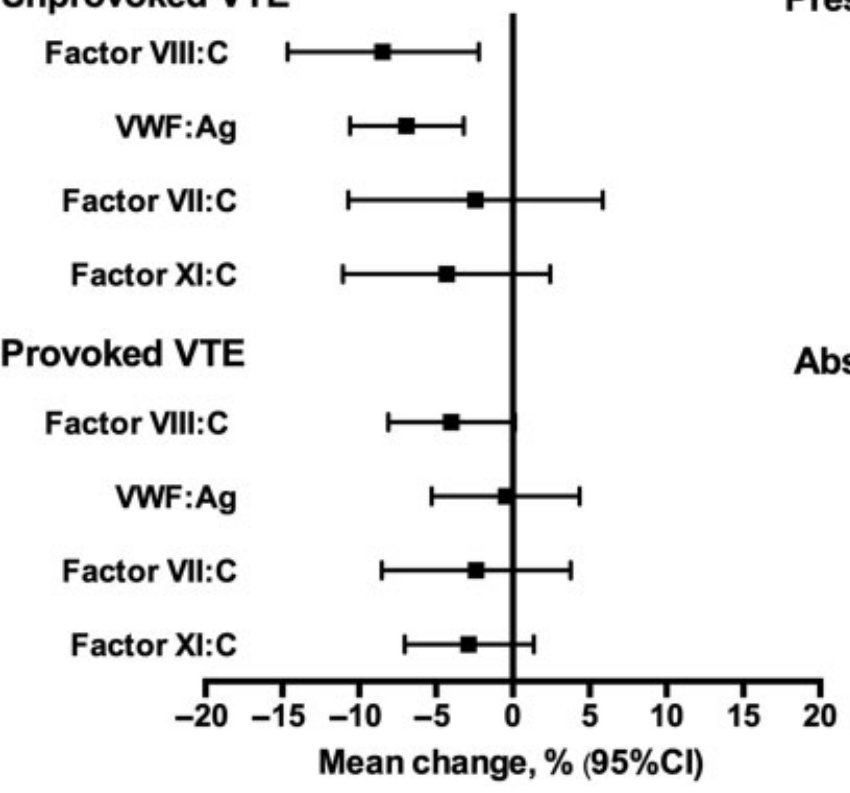

\section{Presence of CV risk factors}

Factor VIII:C

VWF:Ag

Factor VII:C

Factor XI:C

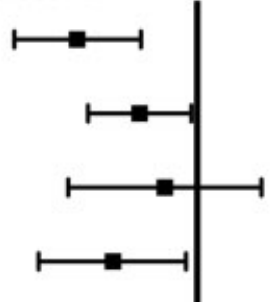

Absence of $\mathrm{CV}$ risk factors

Factor VIII:C

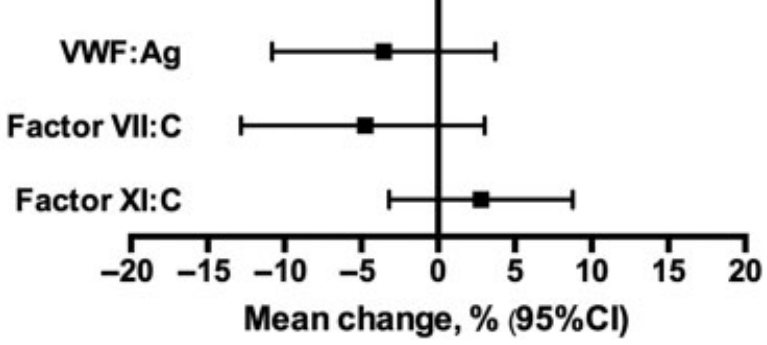

Fig. 1 Effects of rosuvastatin on coagulation factors VIII, XI, VII, VWF, and D-dimer according to (A) VTE classification (unprovoked and provoked) and (B) the presence/absence of cardiovascular risk factors. Results from the START (STAtins Reduce Thrombophilia) trial. ${ }^{63} \mathrm{CI}$, confidence interval; CV, cardiovascular; VTE, venous thromboembolism; VWF, von Willebrand factor. 
atherosclerotic lesions and monocytes independently of the decrease in plasma lipids. ${ }^{43,44}$ The effect of statins on increasing platelet-derived NO release and, in turn, decreasing platelet activity was also shown to be independent of the lipid-lowering effect. ${ }^{26,34}$

In the clinical data, the distinction between a potential direct effect of statin and the lipid-lowering effect on hemostasis is less clear. Most of the studies were conducted in patients with dyslipidemia, and although studies in individuals without dyslipidemia have also demonstrated an effect of statin therapy on hemostasis, ${ }^{54,67,76}$ it is not possible to rule out that the observed effect was due to decreased serum levels of lipids, since many lipoproteins are related to coagulation activity. ${ }^{29}$

\section{The Effect of Statins on the Risk of Venous Thromboembolism}

\section{Evidences from Basic Research Studies}

Data on the effect of statins on in vivo venous thrombosis are limited to few studies. In a mouse model of venous thrombosis, mice treated with high-dose atorvastatin or rosuvastatin displayed $25 \%$ reduction in venous thrombus mass and accelerated thrombus resolution, compared with control animals treated with saline. ${ }^{78}$ The reduction in thrombus formation observed in statin treated mice was accompanied by several changes in platelet activity, fibrinolysis, and coagulation factors. ${ }^{78}$ More recently, a study in a murine model of hyperlipidemic $A P O E$ knockout mice showed that mice treated with high doses of rosuvastatin had a $12 \%$ decrease in venous thrombus formation compared with controls. ${ }^{79}$ The effect of statin on reducing thrombus formation occurred independently of the effect on lipid levels. ${ }^{79}$ Results from these animal studies confirm a biological effect of statins on venous thrombus formation. However, supratherapeutic doses of statins were used in the studies, and doses currently used in the clinical scenario were not tested.

\section{Statins to Prevent First Venous Thromboembolism}

The first study to suggest that statin therapy was associated with a reduced risk of VTE was conducted in a selected population of postmenopausal women. ${ }^{80}$ Since then, several population-based studies have aimed to evaluate the effectiveness of statin therapy on VTE prevention. Most of them are observational studies and were performed in selected populations of patients with CVD. ${ }^{81}$

The only RCT supporting the effect of statins on decreasing VTE risk was published in 2009. In the JUPITER trial, ${ }^{13} 17,802$ apparently healthy individuals, with normal cholesterol levels, were randomized to receive rosuvastatin at $20 \mathrm{mg}$ per day or placebo and were followed for a median period of 1.9 years. Rosuvastatin reduced the incidence of VTE by $40 \%$ (hazard ratio: 0.57 ; 95\% CI: 0.37-0.86) compared with placebo.

Since the results of the JUPITER trial were published, additional evidence of the association between statin therapy and reduced VTE risk came from at least four meta-analyses of observational studies and clinical trials. A meta-analysis of RCTs, published in 2012, compared statin use with no statin
Hazard ratio $(95 \% \mathrm{Cl})$

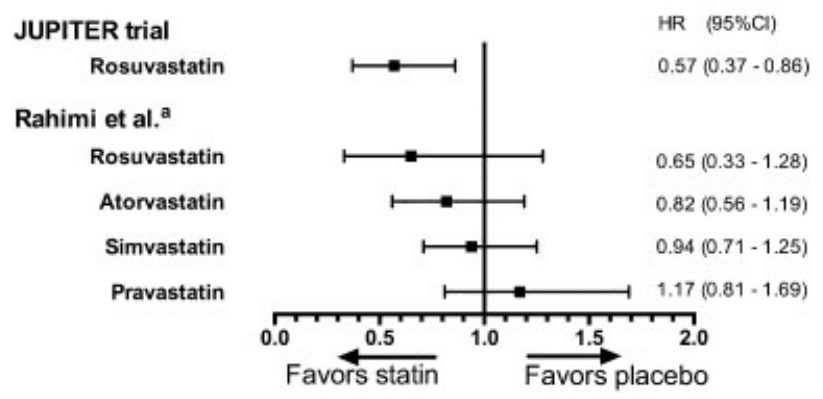

Fig. 2 Effect of rosuvastatin, pravastatin, atorvastatin, and simvastatin on venous thromboembolism. ${ }^{77,78}$ axcluding results from the JUPITER (The Justification for the Use of Statins in Prevention: an Intervention Trial Evaluating Rosuvastatin) trial. Cl, confidence interval; HR, hazard ratio.

(20 trials), and also high dose versus standard dose statin (7 trials). ${ }^{82}$ Results from the pooled analysis did not confirm a risk reduction in VTE by statin treatment. Albeit the CIs were wide, the hazard ratios suggest that rosuvastatin, which is the statin that is most related with halting/regression of atherosclerosis, dyslipidemia, and inflammation, ${ }^{23,83,84}$ also provides the largest risk reductions for the occurrence of VTE. The results are illustrated in -Fig. 2.

A meta-analysis of 36 studies ( 13 cohort studies and 23 RCTs) of statin treatment versus no-statin (placebo or control) was published in 2017. ${ }^{85}$ When evaluating RCTs, the meta-analysis demonstrated a pooled relative risk for VTE of 0.85 (95\% CI: 0.73-0.99) when statin was compared with a nonstatin treatment. The lowest relative risk for VTE was observed in individuals who were randomized to rosuvastatin (relative risk, 0.57 ; 95\% CI: 0.42-0.75). Among observational studies, the pooled relative risk for VTE was 0.75 (95\% CI: 0.65-0.87). A stronger effect, that is, a 54\% risk reduction, was observed in populations with pre-existing disease or at high VTE risk, in contrast with a 14\% VTE risk reduction in studies that recruited participants from the general population. These results are in line with those from a previous meta-analysis of seven observational studies that demonstrated that statin therapy was associated with a $40 \%$ decrease in VTE risk, as compared with no statin use. ${ }^{86}$

Although the results from the JUPITER trial and the metaanalyses of observational studies and RCTs might encourage the use of statins for VTE prevention, these data must be interpreted with caution for several reasons. First, metaanalyses of observational studies have limitations that are related to potential biases in the studies. Some examples are as follows: (1) the underlying disease severity in patients selected for statin therapy may be different, even milder than that in patients for whom statin was not indicated (healthy user effect $)^{87}$ (2) the inclusion of prevalent statin users results in missing the events that occurred in statin users before the inclusion, early after starting treatment (survivor bias); and 3) observational studies are not able to control for those patients who adhere or not to statin treatment (adherence bias). ${ }^{88}$ Second, the meta-analyses of RCTs included nonpublished data and were influenced by the results of the 
JUPITER trial. Finally, the results on VTE from the JUPITER trial came from the analysis of a secondary end-point in the trial, in which the primary outcome was the occurrence of a first major cardiovascular event. These results may have run into the statistical problem of small numbers, since in the statin treatment arm only 34 participants developed a venous thrombotic event, and randomness, or a type I error, may have influenced this result. Replication of the results from the JUPITER trial is important, therefore, to confirm that statin therapy reduces the risk of VTE.

\section{Statins to Prevent Recurrent Venous Thromboembolism}

Over the last years, clinical studies have aimed to determine the role of statins in preventing VTE recurrence, with heterogeneous results. Cohort studies that observed a protective effect of statin therapy on the risk of recurrent VTE have reported a risk reduction that varies from 26 to $38 \% .{ }^{89-91} \mathrm{~A}$ recently published meta-analysis of observational cohort studies demonstrated that the pooled relative risk for VTE recurrence was 0.73 (95\% CI: 0.68-0.79) in statin users in comparison with no use. ${ }^{92}$ Statin therapy reduced the risk for recurrent PE by $25 \%$ and the risk of recurrent DVT by $34 \%$, in comparison with no statin. ${ }^{92}$

A more detailed analysis of the cohort studies, however, reveals that the results may have been affected by the inclusion of long-term (or prevalent) statin users. Long-term statin users may be healthier than nonusers and less susceptible to VTE recurrence, which results in survivor bias and healthy user effect. ${ }^{93}$ As an example, in a large cohort study, Schmidt et al ${ }^{90}$ reported a $29 \%$ decrease (hazard ratio, $95 \% \mathrm{CI}$ : 0.58-0.87) in the risk of recurrent VTE in long-term statin users as compared with nonusers, while among new users the relative risk reduction dropped to a nonsignificant $17 \%(0.45-1.52)$. Therefore, a biased association between statin use and prevention of VTE recurrence cannot be ruled out.

\section{Conclusion}

Several studies have consistently demonstrated that statins affect hemostasis, particularly by downregulating TF expression on endothelium cells and monocytes through a direct, lipidlowering independent effect on transcriptional activation of TF gene. This drug effect is, thus far, the best described mechanism by which statins may reduce thrombus formation. Additionally, statins were also shown to impair platelet activation, decrease multiple coagulation factors, and increase fibrinolysis. A summary with documented biological influence of statins on the different phases of hemostasis is provided in - Fig. 3.

Recent data from randomized trials pointed to a potential effect of statins on decreasing the levels of coagulation factors. Although these studies have provided stronger evidence that statins may affect coagulation in humans, the exact mechanisms by which this happens are not elucidated. Furthermore, the question whether the observed effect of statin therapy on coagulation is sufficient to prevent VTE is still open.

From the clinical perspective, recent meta-analyses of randomized trials have demonstrated that rosuvastatin therapy may reduce the risk of incident VTE, reinforcing the results reported by the JUPITER trial. However, a direct relationship between statin therapy and recurrent VTE risk cannot be assumed from the current clinical data, since the results still need to be replicated. The lack of randomized

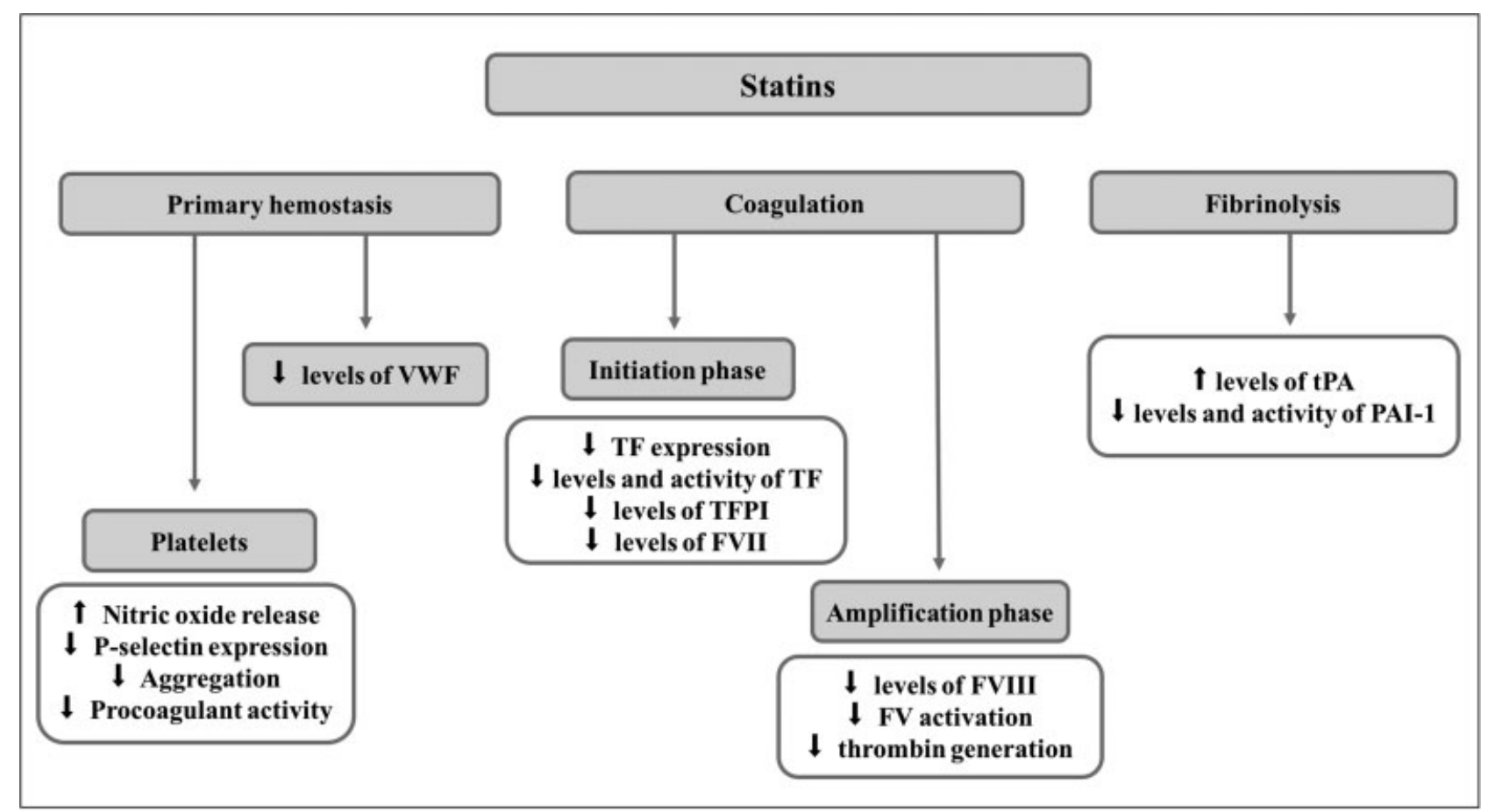

Fig. 3 Biologically documented effects of statins on distinct phases of the process of hemostasis. PAI-1, plasminogen activator inhibitor-1; TFPI, tissue factor pathway inhibitor; tPA, tissue plasminogen activator; VWF, von Willebrand factor. 
data also provides uncertainty about the effect of statin therapy on reducing the risk of recurrent VTE.

Clinical trials are currently underway for assessing whether rosuvastatin (NCT01524653) or simvastatin (NCT02285738) therapy would reduce the risk of incident VTE in patients with cancer. The two studies have surrogate biomarkers of hypercoagulability as a primary endpoints, D-dimer levels, and soluble P-selectin levels, respectively. A third study aims to evaluate whether rosuvastatin can reduce post-thrombotic syndrome in VTE patients (NCT02679664). A fourth trial (NCT02331095) aims to determine whether atorvastatin plus anticoagulation can reduce the thrombin generation potential in patients with acute VTE compared with anticoagulation alone. The results of these trials will contribute to the body of evidence on the association between statin therapy and hemostasis. However, the question whether statin therapy is effective to prevent incident or recurrent VTE will probably remain unanswered for the following years, since no studies on this issue have been started yet.

In conclusion, findings from clinical studies point to a potential effect of statins on decreasing the levels of coagulation factors and reducing the risk for VTE. These effects were mainly attributed to rosuvastatin, which is the most potent LDL-C and atherosclerosis reducing statin. However, stronger evidence on efficacy of statins in preventing VTE, in particular the recurrent events, is still lacking. Recent data showing the effect of rosuvastatin on levels of coagulation factors in patients with prior VTE provides a solid basis for interventional studies necessary to establish the efficacy of statins on reducing the risk of incident of recurrent VTE.

\section{Funding}

F.A.O. received financial support from São Paulo Research Foundation (FAPESP grant\#2017/09506-5).

\section{Conflicts of Interest}

All authors state that they have no conflict of interest.

\section{References}

1 ISTH Steering Committee for World Thrombosis Day. Thrombosis: a major contributor to the global disease burden. J Thromb Haemost 2014;12(10):1580-1590

2 Wendelboe AM, Raskob GE. Global burden of thrombosis: epidemiologic aspects. Circ Res 2016;118(09):1340-1347

3 Kearon C, Ageno W, Cannegieter SC, Cosmi B, Geersing GJ, Kyrle PA; Subcommittees on Control of Anticoagulation, and Predictive and Diagnostic Variables in Thrombotic Disease. Categorization of patients as having provoked or unprovoked venous thromboembolism: guidance from the SSC of ISTH. J Thromb Haemost 2016; 14(07):1480-1483

4 Lijfering WM, Rosendaal FR, Cannegieter SC. Risk factors for venous thrombosis - current understanding from an epidemiological point of view. Br J Haematol 2010;149(06):824-833

5 Kearon C, Akl EA, Ornelas J, et al. Antithrombotic therapy for VTE disease: CHEST guideline and expert panel report. Chest 2016; 149(02):315-352

6 Becattini C, Agnelli G, Schenone A, et al; WARFASA Investigators. Aspirin for preventing the recurrence of venous thromboembolism. N Engl J Med 2012;366(21):1959-1967
7 Agnelli G, Buller HR, Cohen A, et al; AMPLIFY-EXT Investigators. Apixaban for extended treatment of venous thromboembolism. N Engl J Med 2013;368(08):699-708

8 Schulman S, Kearon C, Kakkar AK, et al; RE-MEDY Trial Investigators; RE-SONATE Trial Investigators. Extended use of dabigatran, warfarin, or placebo in venous thromboembolism. N Engl J Med 2013;368(08):709-718

9 Undas A, Brummel-Ziedins KE, Mann KG. Anticoagulant effects of statins and their clinical implications. Thromb Haemost 2014;111 (03):392-400

10 Amarenco P, Bogousslavsky J, Callahan A III, et al; Stroke Prevention by Aggressive Reduction in Cholesterol Levels (SPARCL) Investigators. High-dose atorvastatin after stroke or transient ischemic attack. N Engl J Med 2006;355(06):549-559

11 Collins R, Armitage J, Parish S, Sleight P, Peto R; Heart Protection Study Collaborative Group. Effects of cholesterol-lowering with simvastatin on stroke and other major vascular events in 20536 people with cerebrovascular disease or other high-risk conditions. Lancet 2004;363(9411):757-767

12 Ridker PM, Danielson E, Fonseca FAH, et al; JUPITER Study Group. Rosuvastatin to prevent vascular events in men and women with elevated C-reactive protein. N Engl J Med 2008;359(21):2195-2207

13 Glynn RJ, Danielson E, Fonseca FA, et al. A randomized trial of rosuvastatin in the prevention of venous thromboembolism. $\mathrm{N}$ Engl J Med 2009;360(18):1851-1861

14 Hackam DG, Woodward M, Newby LK, et al. Statins and intracerebral hemorrhage: collaborative systematic review and metaanalysis. Circulation 2011;124(20):2233-2242

15 Morgenstern LB, Hemphill JC III, Anderson C, et al; American Heart Association Stroke Council and Council on Cardiovascular Nursing. Guidelines for the management of spontaneous intracerebral hemorrhage: a guideline for healthcare professionals from the American Heart Association/American Stroke Association. Stroke 2010;41(09):2108-2129

16 Zhang H, Plutzky J, Skentzos S, et al. Discontinuation of statins in routine care settings: a cohort study. Ann Intern Med 2013;158 (07):526-534

17 Stone NJ, Robinson JG, Lichtenstein AH, et al; American College of Cardiology/American Heart Association Task Force on Practice Guidelines. 2013 ACC/AHA guideline on the treatment of blood cholesterol to reduce atherosclerotic cardiovascular risk in adults: a report of the American College of Cardiology/American Heart Association Task Force on Practice Guidelines. J Am Coll Cardiol 2014;63(25 Pt B):2889-2934

18 Mahmoodi BK, Cushman M, Anne Næss I, et al. Association of traditional cardiovascular risk factors with venous thromboembolism: an individual participant data meta-analysis of prospective studies. Circulation 2017;135(01):7-16

19 Zöller B, Li X, Sundquist J, Sundquist K. Socioeconomic and occupational risk factors for venous thromboembolism in Sweden: a nationwide epidemiological study. Thromb Res 2012;129 (05):577-582

20 Abdollahi M, Cushman M, Rosendaal FR. Obesity: risk of venous thrombosis and the interaction with coagulation factor levels and oral contraceptive use. Thromb Haemost 2003;89(03):493-498

21 Roach RE, Lijfering WM, Flinterman LE, Rosendaal FR, Cannegieter SC. Increased risk of CVD after VT is determined by common etiologic factors. Blood 2013;121(24):4948-4954

22 Vaughan CJ, Gotto AM Jr, Basson CT. The evolving role of statins in the management of atherosclerosis. J Am Coll Cardiol 2000;35 (01):1-10

23 Ridker PM, Danielson E, Fonseca FA, et al; JUPITER Trial Study Group. Reduction in C-reactive protein and LDL cholesterol and cardiovascular event rates after initiation of rosuvastatin: a prospective study of the JUPITER trial. Lancet 2009;373(9670):1175-1182

24 Schäfer A, Fraccarollo D, Eigenthaler M, et al. Rosuvastatin reduces platelet activation in heart failure: role of NO bioavailability. Arterioscler Thromb Vasc Biol 2005;25(05):1071-1077 
25 Gertz K, Laufs U, Lindauer U, et al. Withdrawal of statin treatment abrogates stroke protection in mice. Stroke 2003;34(02):551-557

26 Laufs U, Gertz K, Huang P, et al. Atorvastatin upregulates type III nitric oxide synthase in thrombocytes, decreases platelet activation, and protects from cerebral ischemia in normocholesterolemic mice. Stroke 2000;31(10):2442-2449

27 Ni R, Peleg T, Gross PL. Atorvastatin delays murine platelet activation in vivo even in the absence of endothelial NO synthase. Arterioscler Thromb Vasc Biol 2012;32(11):2609-2615

28 Hamada M, Sugimoto M, Matsui H, et al. Antithrombotic properties of pravastatin reducing intra-thrombus fibrin deposition under high shear blood flow conditions. Thromb Haemost 2011;105(02):313-320

29 Lippi G, Franchini M, Targher G. Arterial thrombus formation in cardiovascular disease. Nat Rev Cardiol 2011;8(09):502-512

30 Aoki I, Aoki N, Kawano K, et al. Platelet-dependent thrombin generation in patients with hyperlipidemia. J Am Coll Cardiol 1997;30(01):91-96

31 Puccetti L, Pasqui AL, Pastorelli M, et al. Time-dependent effect of statins on platelet function in hypercholesterolaemia. Eur J Clin Invest 2002;32(12):901-908

32 Dujovne CA, Harris WS, Altman R, Overhiser RW, Black DM. Effect of atorvastatin on hemorheologic-hemostatic parameters and serum fibrinogen levels in hyperlipidemic patients. Am J Cardiol 2000;85(03):350-353

33 Puccetti L, Pasqui AL, Pastorelli M, et al. Platelet hyperactivity after statin treatment discontinuation. Thromb Haemost 2003;90(03): 476-482

34 Haramaki N, Ikeda H, Takenaka K, et al. Fluvastatin alters platelet aggregability in patients with hypercholesterolemia: possible improvement of intraplatelet redox imbalance via HMG-CoA reductase. Arterioscler Thromb Vasc Biol 2007;27(06):1471-1477

35 Ural AU, Yilmaz MI, Avcu F, Yalcin A. Treatment with cerivastatin in primary mixed hyperlipidemia induces changes in platelet aggregation and coagulation system components. Int J Hematol 2002;76(03):279-283

36 Ali FY, Armstrong PC, Dhanji AR, et al. Antiplatelet actions of statins and fibrates are mediated by PPARs. Arterioscler Thromb Vasc Biol 2009;29(05):706-711

37 Biedermann JS, Cannegieter SC, Roest M, et al. Platelet reactivity in patients with venous thrombosis who use rosuvastatin: a randomized controlled clinical trial. J Thromb Haemost 2016; 14(07):1404-1409

38 Colli S, Eligini S, Lalli M, Camera M, Paoletti R, Tremoli E. Vastatins inhibit tissue factor in cultured human macrophages. A novel mechanism of protection against atherothrombosis. Arterioscler Thromb Vasc Biol 1997;17(02):265-272

39 Eto M, Kozai T, Cosentino F, Joch H, Lüscher TF. Statin prevents tissue factor expression in human endothelial cells: role of Rho/Rhokinase and Akt pathways. Circulation 2002;105(15):1756-1759

40 Ferro D, Basili S, Alessandri C, Cara D, Violi F. Inhibition of tissuefactor-mediated thrombin generation by simvastatin. Atherosclerosis 2000;149(01):111-116

41 Sen-Banerjee S, Mir S, Lin Z, et al. Kruppel-like factor 2 as a novel mediator of statin effects in endothelial cells. Circulation 2005; 112(05):720-726

42 Aikawa M, Rabkin E, Sugiyama S, et al. An HMG-CoA reductase inhibitor, cerivastatin, suppresses growth of macrophages expressing matrix metalloproteinases and tissue factor in vivo and in vitro. Circulation 2001;103(02):276-283

43 Baetta R, Camera M, Comparato C, Altana C, Ezekowitz MD, Tremoli E. Fluvastatin reduces tissue factor expression and macrophage accumulation in carotid lesions of cholesterol-fed rabbits in the absence of lipid lowering. Arterioscler Thromb Vasc Biol 2002;22(04):692-698

44 Bea F, Blessing E, Shelley MI, Shultz JM, Rosenfeld ME. Simvastatin inhibits expression of tissue factor in advanced atherosclerotic lesions of apolipoprotein $\mathrm{E}$ deficient mice independently of lipid lowering: potential role of simvastatin-mediated inhibition of Egr-1 expression and activation. Atherosclerosis 2003;167(02): 187-194

45 Nagata K, Ishibashi T, Sakamoto T, et al. Rho/Rho-kinase is involved in the synthesis of tissue factor in human monocytes. Atherosclerosis 2002;163(01):39-47

46 Camera M, Toschi V, Comparato C, et al. Cholesterol-induced thrombogenicity of the vessel wall: inhibitory effect of fluvastatin. Thromb Haemost 2002;87(04):748-755

47 Szczeklik A, Musiał J, Undas A, et al. Inhibition of thrombin generation by simvastatin and lack of additive effects of aspirin in patients with marked hypercholesterolemia. J Am Coll Cardiol 1999;33(05):1286-1293

48 Cortellaro M, Cofrancesco E, Arbustini E, et al. Atorvastatin and thrombogenicity of the carotid atherosclerotic plaque: the ATROCAP study. Thromb Haemost 2002;88(01):41-47

49 Morishita E, Minami S, Ishino C, et al. Atorvastatin reduces plasma levels of factor VII activity and factor VII antigen in patients with hyperlipidemia. J Atheroscler Thromb 2002;9(01):72-77

50 Porreca E, Di Febbo C, Amore C, et al. Effect of lipid-lowering treatment on factor VII profile in hyperlipidemic patients. Thromb Haemost 2000;84(05):789-793

51 Morishita E, Asakura H, Saito M, et al. Elevated plasma levels of free-form of TFPI antigen in hypercholesterolemic patients. Atherosclerosis 2001;154(01):203-212

52 Ferro D, Basili S, Alessandri C, Mantovani B, Cordova C, Violi F. Simvastatin reduces monocyte-tissue-factor expression type IIa hypercholesterolaemia. Lancet 1997;350(9086):1222

53 Krysiak R, Okopien B. Effect of simvastatin on hemostasis in patients with isolated hypertriglyceridemia. Pharmacology 2013;92(3-4):187-190

54 Steiner S, Speidl WS, Pleiner J, et al. Simvastatin blunts endotoxininduced tissue factor in vivo. Circulation 2005;111(14):1841-1846

55 Tousoulis D, Bosinakou E, Kotsopoulou M, Antoniades C, Katsi V, Stefanadis C. Effects of early administration of atorvastatin treatment on thrombotic process in normocholesterolemic patients with unstable angina. Int J Cardiol 2006;106(03):333-337

56 Undas A, Brummel KE, Musial J, Mann KG, Szczeklik A. Simvastatin depresses blood clotting by inhibiting activation of prothrombin, factor V, and factor XIII and by enhancing factor Va inactivation. Circulation 2001;103(18):2248-2253

57 Undas A, Celinska-Löwenhoff M, Brummel-Ziedins KE, Brozek J, Szczeklik A, Mann KG. Simvastatin given for 3 days can inhibit thrombin generation and activation of factor $V$ and enhance factor $\mathrm{Va}$ inactivation in hypercholesterolemic patients. Arterioscler Thromb Vasc Biol 2005;25(07):1524-1525

58 Joukhadar C, Klein N, Prinz M, et al. Similar effects of atorvastatin, simvastatin and pravastatin on thrombogenic and inflammatory parameters in patients with hypercholesterolemia. Thromb Haemost 2001;85(01):47-51

59 Ordulu E, Erdogan O. Early effects of low versus high dose atorvastatin treatment on coagulation and inflammation parameters in patients with acute coronary syndromes. Int J Cardiol 2008;128(02):282-284

60 Musiał J, Undas A, Undas R, Brozek J, Szczeklik A. Treatment with simvastatin and low-dose aspirin depresses thrombin generation in patients with coronary heart disease and borderline-high cholesterol levels. Thromb Haemost 2001;85(02):221-225

61 Cipollone F, Mezzetti A, Porreca E, et al. Association between enhanced soluble CD40L and prothrombotic state in hypercholesterolemia: effects of statin therapy. Circulation 2002;106(04):399-402

62 Sanguigni V, Pignatelli P, Lenti L, et al. Short-term treatment with atorvastatin reduces platelet CD40 ligand and thrombin generation in hypercholesterolemic patients. Circulation 2005;111(04):412-419

63 Kaba NK, Francis CW, Moss AJ, et al; THROMBO Investigators. Effects of lipids and lipid-lowering therapy on hemostatic factors in patients with myocardial infarction. J Thromb Haemost 2004;2 (05):718-725 
64 Tonkin AM, Blankenberg S, Kirby A, et al; LIPID study investigators. Biomarkers in stable coronary heart disease, their modulation and cardiovascular risk: The LIPID biomarker study. Int J Cardiol 2015;201:499-507

65 Sahebkar A, Serban C, Ursoniu S, et al; Lipid and Blood Pressure Meta-analysis Collaboration (LBPMC) Group. The impact of statin therapy on plasma levels of von Willebrand factor antigen. Systematic review and meta-analysis of randomised placebocontrolled trials. Thromb Haemost 2016;115(03):520-532

66 Sahebkar A, Serban C, Mikhailidis DP, et al; Lipid and Blood Pressure Meta-analysis Collaboration (LBPMC) Group. Association between statin use and plasma D-dimer levels. A systematic review and meta-analysis of randomised controlled trials. Thromb Haemost 2015;114(03):546-557

67 Biedermann JS, Kruip MJHA, van der Meer FJ, et al. Rosuvastatin use improves measures of coagulation in patients with venous thrombosis. Eur Heart J 2018;39(19):1740-1747

68 Bevilacqua M, Bettica P, Milani M, et al. Effect of fluvastatin on lipids and fibrinolysis in coronary artery disease. Am J Cardiol 1997;79(01):84-87

69 Dangas G, Badimon JJ, Smith DA, et al. Pravastatin therapy in hyperlipidemia: effects on thrombus formation and the systemic hemostatic profile. J Am Coll Cardiol 1999;33(05):1294-1304

70 Dangas G, Smith DA, Unger AH, et al. Pravastatin: an antithrombotic effect independent of the cholesterol-lowering effect. Thromb Haemost 2000;83(05):688-692

71 Bruni F, Pasqui AL, Pastorelli M, et al. Effect of atorvastatin on different fibrinolyis mechanisms in hypercholesterolemic subjects. Int J Cardiol 2004;95(2-3):269-274

72 Tetik S, Ak K, Sahin Y, et al. Postoperative statin therapy attenuates the intensity of systemic inflammation and increases fibrinolysis after coronary artery bypass grafting. Clin Appl Thromb Hemost 2011;17(05):526-531

73 Ushiroyama T, Nosaka S, Ueki M. Short-term effects of low-dose atorvastatin on inflammatory status and lipid profiles in perimenopausal hypercholesterolemic, hypertriglyceridemic women. Int J Cardiol 2006;113(01):66-75

74 Liu L, Zhao SP, Zhou HN, Li QZ, Li JX. Effect of fluvastatin and valsartan, alone and in combination, on postprandial vascular inflammation and fibrinolytic activity in patients with essential hypertension. J Cardiovasc Pharmacol 2007;50(01):50-55

75 Undas A, Kaczmarek P, Sladek K, et al. Fibrin clot properties are altered in patients with chronic obstructive pulmonary disease. Beneficial effects of simvastatin treatment. Thromb Haemost 2009;102(06):1176-1182

76 Adams NB, Lutsey PL, Folsom AR, et al. Statin therapy and levels of hemostatic factors in a healthy population: the multi-ethnic study of atherosclerosis. J Thromb Haemost 2013;11(06): 1078-1084

77 Sahebkar A, Catena C, Ray KK, et al. Impact of statin therapy on plasma levels of plasminogen activator inhibitor- 1 . A systematic review and meta-analysis of randomised controlled trials. Thromb Haemost 2016;116(01):162-171

78 Kessinger CW, Kim JW, Henke PK, et al. Statins improve the resolution of established murine venous thrombosis: reductions in thrombus burden and vein wall scarring. PLoS One 2015;10 (02):e0116621

79 Patterson KA, Zhang X, Wrobleski SK, et al. Rosuvastatin reduced deep vein thrombosis in ApoE gene deleted mice with hyperlipidemia through non-lipid lowering effects. Thromb Res 2013;131 (03):268-276

80 Grady D, Wenger NK, Herrington D, et al. Postmenopausal hormone therapy increases risk for venous thromboembolic disease. The heart and estrogen/progestin replacement study. Ann Intern Med 2000;132(09):689-696

81 Lijfering WM, Biedermann JS, Kruip MJ, Leebeek FW, Rosendaal FR, Cannegieter SC. Can we prevent venous thrombosis with statins: an epidemiologic review into mechanism and clinical utility. Expert Rev Hematol 2016;9(11):1023-1030

82 Rahimi K, Bhala N, Kamphuisen P, et al. Effect of statins on venous thromboembolic events: a meta-analysis of published and unpublished evidence from randomised controlled trials. PLoS Med 2012;9(09):e1001310

83 Law MR, Wald NJ, Rudnicka AR. Quantifying effect of statins on low density lipoprotein cholesterol, ischaemic heart disease, and stroke: systematic review and meta-analysis. BMJ 2003;326(7404):1423

84 Nissen SE, Nicholls SJ, Sipahi I, et al; ASTEROID Investigators. Effect of very high-intensity statin therapy on regression of coronary atherosclerosis: the ASTEROID trial. JAMA 2006;295 (13):1556-1565

85 Kunutsor SK, Seidu S, Khunti K. Statins and primary prevention of venous thromboembolism: a systematic review and meta-analysis. Lancet Haematol 2017;4(02):e83-e93

86 Pai M, Evans NS, Shah SJ, Green D, Cook D, Crowther MA. Statins in the prevention of venous thromboembolism: a meta-analysis of observational studies. Thromb Res 2011;128(05):422-430

87 Thomsen RW. The lesser known effects of statins: benefits on infectious outcomes may be explained by "healthy user" effect. BMJ 2006;333(7576):980-981

88 van Rein N, Cannegieter SC, le Cessie S, et al. Statins and risk of bleeding: an analysis to evaluate possible bias due to prevalent users and healthy user aspects. Am J Epidemiol 2016;183(10): 930-936

89 Nguyen CD, Andersson C, Jensen TB, et al. Statin treatment and risk of recurrent venous thromboembolism: a nationwide cohort study. BMJ Open 2013;3(11):e003135

90 Schmidt M, Cannegieter SC, Johannesdottir SA, Dekkers OM, Horváth-Puhó E, Sørensen HT. Statin use and venous thromboembolism recurrence: a combined nationwide cohort and nested case-control study. J Thromb Haemost 2014;12(08):1207-1215

91 Smith NL, Harrington LB, Blondon M, et al. The association of statin therapy with the risk of recurrent venous thrombosis. J Thromb Haemost 2016;14(07):1384-1392

92 Kunutsor SK, Seidu S, Khunti K. Statins and secondary prevention of venous thromboembolism: pooled analysis of published observational cohort studies. Eur Heart J 2017;38(20):1608-1612

93 Brookhart MA, Patrick AR, Dormuth C, et al. Adherence to lipidlowering therapy and the use of preventive health services: an investigation of the healthy user effect. Am J Epidemiol 2007;166 (03):348-354 